\title{
COMPLETE EMBEDDED MINIMAL SURFACES OF FINITE TOTAL CURVATURE
}

\author{
BY DAVID A. HOFFMAN ${ }^{1}$ AND WILLIAM H. MEEKS III $^{2}$
}

A long-standing problem in the theory of minimal surfaces is the construction of complete embedded minimal surfaces with finite topology. Many examples of complete properly embedded minimal surfaces in Euclidean threespace have been constructed, but except for the plane, the catenoid, and the helicoid, all the known surfaces have infinite genus. Since it is natural to try to develop a theory of the global geometry of embedded minimal surfaces of finite type, the lack of examples is a major obstacle. In fact, it has often been conjectured that these three examples are the only complete embedded minimal surfaces in $\mathbf{R}^{3}$ of finite topological type.

In 1980, Jorge and Meeks [3] developed a theory to study the topology of complete embedded minimal surfaces in $\mathbf{R}^{3}$ of finite total curvature. (By a classical theorem of $\mathrm{R}$. Osserman [4], a complete minimal surface of finite total curvature is conformally a compact Riemann surface with a finite number of points removed.) They were able to prove that there were no complete embedded minimal surfaces of finite total curvature of genus zero with three, four, or five ends, and, except for the plane, an embedded complete minimal surface of finite total curvature had at least two ends. Recently, R. Schoen [5] has proved that the only complete embedded minimal surface of any genus with finite total curvature and two ends is the catenoid. The catenoid has genus zero, two ends, and total curvature $-4 \pi$.

We have found that there exist embedded minimal surfaces of finite total curvature of every genus.

THEOREM 1. For every genus $g>0$ there exists a complete embedded minimal surface $M_{g}$ of genus $g$ with three ends and finite total curvature $-4 \pi(g+2)$.

Using this theorem we have the following corollary.

COROLlaRY. For every nonnegative integer $k$, except $k=2$, there exists a complete embedded minimal surface with total curvature equal to $-4 k \pi$.

PROOF OF COROLlARY. The examples of Theorem 1, together with the plane and the catenoid, give examples of complete embedded minimal surfaces of total curvature $C=-4 \pi k$ for every nonnegative integer $k$ except $k=2$. In $[3]$ it is proved that on a complete embedded minimal surface of finite total curvature, $C=-4 \pi(g+r-1)$, where $g$ is the genus and $r \geq 1$ is the number

Received by the editors August 6, 1984 and, in revised form, October 4, 1984.

1980 Mathematics Subject Classification. Primary 53A10, 49F10, 58E12.

${ }^{1}$ Supported by DMS Grant MCS- 8301936 .

${ }^{2}$ Supported by NSF Grant DMS-84-14330. 
of ends. If $C=-8 \pi, g+r=3$ with $r \geq 1$. By the results of Schoen and Jorge and Meeks mentioned earlier, this is not possible.

We have constructed the examples of Theorem 1 using the Weierstrass representation formulae, giving an explicit conformal immersion of the surface in terms of meromorphic functions. In the case of genus one our surface is the example of Costa $[\mathbf{1}]$, which is constructed using the Weierstrass $\wp$-function and its derivative on the square lattice. Costa proved that the surface was complete, and it followed from the results of Jorge and Meeks that the surface was embedded outside of a compact ball. Our work was motivated by this example. In particular, we used a computer to explicitly determine the coordinate functions of this surface and draw it. From the pictures we could see that the surface was embedded, possessed dihedral symmetry, and contained two orthogonal lines. We then found mathematical proofs of these observations. Our examples of higher genus are constructed to possess dihedral symmetry and contain straight lines, properties upon which the mathematical proof of embeddedness is based.

THEOREM 2. The surface $M_{g}$ has the following properties.

(i) Its symmetry group is the dihedral group of order $4(g+1)$ generated by reflection in the $(x, z)$-plane and by a rotation of $\pi$ radians about the line in the $(x, y)$-plane making a positive angle of $\pi / 2(g+1)$ radians with the $x$-axis.

(ii) The intersection of the $(x, y)$-plane with $M_{g}$ consists of $g+1$ straight lines meeting at equal angles at the origin. The planes $z=c \neq 0$ intersect the surface in a single Jordan curve.

(iii) $M_{g}$ is the unique embedded minimal surface of genus $g$ with three ends, finite total curvature, and symmetry group containing at least $4(g+1)$ elements.

The surface $M_{g}$ is constructed from the classical Riemann surface where the function $y$, given by the equation $y^{(g+1)}=z^{g}\left(z^{2}-1\right)$, is well defined, by removing the points where $z$ equals $1,-1$, and $\infty$. In terms of the Weierstrass representation (see, for example, $[3]$ ), the meromorphic functions on $M_{g}$ are constructed from the functions $z$ and $y$. Specifically, the Gauss map composed with stereographic projection is equal to a real constant divided by $y$, and the auxiliary one-form $\eta$ is given by $\eta=(z / y)^{g} d z$.

These and other results will be published elsewhere.

ACKNOWLEDGEMENTS. We wish to thank J. Hoffman for critical assistance in computer graphics programming and the Research Computing Facility of the Computer Science Department at the University of Massachusetts for the use of their facilities. For helpful discussions we wish to thank P. Norman, R. Osserman, and R. Schoen.

\section{REFERENCES}

1. C. Costa, Imersões mınımas completas em $\mathbf{R}^{3}$ de gênero um e curvatura total finita, Doctoral thesis, IMPA, Rio de Janeiro, Brasil, 1982.

2. D. Hoffman and R. Osserman, The geometry of the generalized Gauss map, Mem. Amer. Math. Soc. No. 236 (1980). 
3. L. Jorge and W. Meeks III, The topology of complete minimal surfaces of finite total Gaussian curvature, Topology 22 (1983), 203-221.

4. R. Osserman, Global properties of complete minimal surfaces in $E^{3}$ and $E^{n}$, Ann. of Math. (2) 80 (1964), 340-364.

5. R. Schoen, Uniqueness, symmetry, and embeddedness of minimal surfaces, J. Differential Geom. 18 (1983), 791-809.

DEPARTMENT OF MATHEMATICS AND STATISTICS, UNIVERSity OF MASSAChusetts, Lederle Graduate Research Center Towers, Amherst, MassaCHUSETTS 01003

Department of Mathematics, Rice University, Houston, Texas 77001 